\title{
Frames from Cosines with the Degenerate Coefficients
}

\author{
Sadigova Sabina Rahib*, Mamedova Zahira Vahid \\ Institute of Mathematics and Mechanics of NAS of Azerbaijan, B.Vahabzade 9, AZ1141,Baku, Azerbaijan \\ *Corresponding author: s_sadigova@ mail.ru
}

Received December 23, 2012; Revised May 09, 2013; Accepted May 10, 2013

\begin{abstract}
The system of cosines with a degenerate coefficient in exponential form is considered. A necessary and sufficient condition on the degree of degeneration is found that makes the considered system a frame in Lebesgue spaces. It is proved that if the degenerate coefficient satisfies the Muckenhoupt condition, then the basicity holds. If the Muckenhoupt condition does not hold, then the system has a finite defect, and does not form a frame.
\end{abstract}

Keywords: systems of cosines, degeneration, frames

\section{Introduction}

Basis properties of classical system of exponents $\left\{e^{\text {int }}\right\}_{n \in Z}$ ( $Z$ is the set of all integers) in Lebesgue spaces $L_{p}(-\pi, \pi), 1 \leq p<+\infty$, are well studied in the literature (see $[6,7,17,18,19])$. N.K.Bari in her fundamental work [2] raised the issue of the existence of normalized basis in $L_{2}$ which is not Riesz basis. The first example of this was given by K.I.Babenko [1]. He proved that the degenerate system of exponents $\left\{|x|^{\alpha} e^{\text {int }}\right\}_{n \in Z}$ with $|\alpha|<\frac{1}{2}$ forms a basis for $L_{2}(-\pi, \pi)$ but is not Riesz basis when $\alpha \neq 0$. This result has been extended by V.F.Gaposhkin [8]. In [13], the condition on the weight $\rho$ was found which make the system $\left\{e^{\text {int }}\right\}_{n \in Z}$ form a basis for the weight space $L_{p, \rho}(-\pi, \pi)$ with a norm $\|f\|_{p, \rho}=\left(\int_{-\pi}^{\pi}|f(t)|^{p} \rho(t) d t\right)^{\frac{1}{p}}$.

Basis properties of a degenerate system of exponents are closely related to the similar properties of an ordinary system of exponents in corresponding weight space. In all the mentioned works the authors consider the cases when the weight or the degenerate coefficient satisfies the Muckenhoupt condition (see, for example, [9]). It should be noted that the above-stated is true for the systems of sines and cosines, too.

Basis properties of the system of exponents and sines with the linear phase in weighted Lebesgue spaces have been studied in $[14,15,16]$. Those of thpe systems of exponents with degenerate coefficients have been studied in $[3,4]$.

In this work, we study the frame properties of the system of cosines with degenerate coefficients in Lebesgue spaces. Similar problems have previously been considered in paper $[11,12]$.

\section{Needful Information}

To obtain our main results, we will use some concepts and facts from the theory of bases.

We will use the standard notation. $N$ will be the set of all positive integers, $\exists$ will mean "there exist(s)", $\Rightarrow$ will mean "it follows", $\Leftrightarrow$ will mean "if and only if", $\exists$ ! will mean "there exists unique", $K \equiv R$ or $K \equiv C$ will stand for the set of real or complex numbers, respectively.

Let $X$ be some Banach space with a norm $\|\cdot\|_{X}$. Then $X^{*}$ will denote its conjugate with a norm $\|\cdot\|_{X}{ }^{*}$. By $L[M]$ we denote the linear span of the set $M \subset X$, and $\bar{M}$ will stand for the closure of $M$.

System $\left\{x_{n}\right\}_{n \in N} \subset X$ is said to be complete in $X$ if $\overline{L\left[\left\{x_{n}\right\}_{n \in N}\right]}=X$. It is called minimal in $X$ if $x_{k} \notin \overline{L\left[\left\{x_{n}\right\}_{n \neq k}\right]}, \forall k \in N$.

System $\left\{x_{n}\right\}_{n \in N} \subset X$ is said to be uniformly minimal in $X$ if $\exists \delta>0$ :

$$
\inf _{\forall u \in L\left[\left\{x_{n}\right\}_{n \neq k}\right]}\left\|x_{k}-u\right\|_{X} \geq \delta\left\|x_{k}\right\|_{X}, \quad \forall k \in N .
$$

The following criteria of completeness and minimality are available:

Criterion 1. System $\left\{x_{n}\right\}_{n \in N} \subset X$ is complete in $X$ if $f\left(x_{n}\right)=0, \forall n \in N, f \in X^{*} \Rightarrow f=0$.

Criterion 2. System $\left\{x_{n}\right\}_{n \in N} \subset X$ is minimal in $X \Leftrightarrow$ it has a biorthogonal system $\left\{f_{n}\right\}_{n \in N} \subset X^{*}$, i.e. $f_{n}\left(x_{k}\right)=\delta_{n k}, \forall n, k \in N, \quad$ where $\delta_{n k}$ is the Kronecker symbol. 
Criterion 3. Complete system $\left\{x_{n}\right\}_{n \in N} \subset X$ is uniformly minimal in $X \Leftrightarrow \sup _{n}\left\|x_{n}\right\|_{X}\left\|y_{n}\right\|_{X^{*}<+\infty}$, where $\left\{y_{n}\right\}_{n \in N} \subset X^{*}$ is a system biorthogonal to it.

System $\left\{x_{n}\right\}_{n \in N} \subset X$ is said to be a basis for $X$ if $\forall x \in X, \exists !\left\{\lambda_{n}\right\}_{n \in N} \subset K: x=\sum_{n=1}^{\infty} \lambda_{n} x_{n}$.

System $\left\{x_{n}\right\}_{n \in N} \subset X$ is said to be a frame if $\forall x \in \overline{L\left[\left\{x_{n}\right\}_{n \in N}\right]}, \exists\left\{\lambda_{n}\right\}_{n \in N} \subset K: x=\sum_{n=1}^{\infty} \lambda_{n} x_{n}$.

If system $\left\{x_{n}\right\}_{n \in N} \subset X$ forms a basis for $X$, then it is uniformly minimal.

More details about these facts can be found in $[5,10,12]$.

\section{Completeness and Minimality}

We consider a system of cosines

$$
\left\{c_{n}^{\omega}\right\}_{n \in Z_{+}} \equiv\{\omega(t) \cos n t\}_{n \in Z_{+}}
$$

$\left(Z_{+} \equiv 0 ; 1 ; \ldots\right)$ with a degenerate coefficient $\omega$ :

$$
\omega(t)=t^{\alpha_{0}} \prod_{k=0}^{r}\left|t-t_{k}\right|^{\alpha_{k}}
$$

where $\left\{t_{k}\right\}_{1}^{r} \subset(0, \pi]: t_{i} \neq t_{j}$ for $i \neq j$. The symbol $f \sim g, t \rightarrow a \quad$, means that in sufficiently small neighborhood of the point $t=a$ there holds the inequality $0<\delta \leq\left|\frac{f(t)}{g(t)}\right| \leq \delta^{-1}<+\infty$.

Theorem 1. Let the conditions

$$
\left\{\alpha_{k}\right\}_{0}^{r} \subset\left(-\frac{1}{p},+\infty\right)
$$

be satisfied. Then the system $\left\{c_{n}^{\omega}\right\}_{n \in Z_{+}}$is complete in $L_{p}, 1 \leq p<+\infty$. If the following relation holds

$$
\left\{\alpha_{k}\right\}_{0}^{r} \subset\left(-\frac{1}{p}, \frac{1}{q}\right),
$$

then for $p \in(1,+\infty)$ it forms a basis for $L_{p}$, but in a case $\left\{\alpha_{k}\right\}_{0}^{r} \subset(-1,0]$ this system is complete and minimal in $L_{1}$, but does not form a basis for it.

Proof. Thus, it is clear that the system $\left\{c_{n}^{\omega}\right\}_{n \in Z_{+}}$ belongs to the space $L_{p} \equiv L_{p}(0, \pi), 1 \leq p<+\infty$, if and only if the relation (1) holds.

Let us consider the completeness of this system. Let $f \in L_{q},\left(\frac{1}{p}+\frac{1}{q}=1\right)$ cancels the system $\left\{c_{n}^{\omega}\right\}_{n \in Z_{+}}$out, that is

$$
\left\langle c_{n}^{\omega}, f\right\rangle \equiv \int_{0}^{\pi} c_{n}^{\omega} \overline{f(t)} d t=0, \forall n \in Z_{+}
$$

where $(\stackrel{\cdot}{\cdot})$ is a complex conjugate. By $C[0, \pi]$ we denote the Banach space of functions which are continuous on $[0, \pi]$ with a sup -norm. It is absolutely clear that $\omega f \in L_{1}(0, \pi) \subset C^{*}[0, \pi]$. As the system of cosines $\{\cos n t\}_{n \in Z_{+}}$is complete in $C[0, \pi]$, we obtain from the relations (2) that $\omega f=0 \Rightarrow f=0$. This proves the completeness of system $\left\{c_{n}^{\omega}\right\}_{n \in Z_{+}}$in $L_{p}(0, \pi)$. Now consider the minimality of this system in $L_{p}$. It is clear that the system $\left\{c_{n}^{\omega^{-1}}\right\}_{n \in Z_{+}}$belongs to $L_{q}$ if and only if

$$
\left\{\alpha_{k}\right\}_{0}^{r} \subset\left(-\infty, \frac{1}{q}\right)
$$

The theorem is proved.

It should be noted that these facts can be directly obtained from the classical results.

\section{Defective Case}

Here we consider the defective system of cosines $\left\{c_{n}^{\omega}\right\}_{n \in N_{\left(k_{0}\right)}}$, where $N_{\left(k_{0}\right)} \equiv Z_{+} \backslash\left\{k_{0}\right\}, k_{0} \in Z_{+}$is some number.

The following theorem is true.

Theorem 2. Let the necessary condition $\alpha_{0} \in\left(-\frac{1}{p},+\infty\right),\left\{\alpha_{k}\right\}_{1}^{r} \subset\left(-\frac{1}{p} ; \frac{1}{q}\right)$ be satisfied. Then the system $\left\{c_{n}^{\omega}\right\}_{n \in Z_{+}}$is a frame (basis) in $L_{p}$ if and only if $\alpha_{0} \in\left(-\frac{1}{p}, \frac{1}{q}\right)$. Moreover, for $\alpha_{0} \in M_{p}^{(k)}, k \in N$, it has a defect equal to $(k)$, where $M_{p}^{(k)} \equiv\left[\frac{1}{q}+2(k-1), \frac{1}{q}+2 k\right)$. Assume

$$
\alpha_{0} \in M_{q}^{(1)} \equiv\left[\frac{1}{q}, \frac{1}{q}+2\right)
$$

$\left\{\alpha_{k}\right\}_{1}^{r} \subset\left(-\frac{1}{p}, \frac{1}{q}\right)$. Suppose that $f \in L_{q}$ cancels system $\left\{c_{n}^{\omega}\right\}_{n \in N_{\left(k_{0}\right)}}$ out, that is, $\left\langle c_{n}^{\omega}, f\right\rangle=0$, $\forall n \in N_{\left(k_{0}\right)} \cdot$

Consequently,

$$
\langle\cos n t, \omega f\rangle=0, \forall n \in N_{\left(k_{0}\right)}
$$

As $\omega f \in L_{1} \subset C^{*}[0, \pi]$ and system $\{\cos n t\}_{n \in Z_{+}}$is complete and minimal in $C[0, \pi]$, from (4) we get 
$\omega f=c \cos k_{0} t$, where $c$ is some constant. Then from expression $f=c \omega^{-1} \cos k_{0} t$ follows that $f \in L_{q}$ if and only if $c=0$. Thus, system $\left\{c_{n}^{\omega}\right\}_{n \in N}\left(k_{0}\right)$ is complete in $L_{p}$.

Now we consider the minimality of this system. Let

$$
\vartheta_{n}(t)=\omega^{-1}(t)\left(\cos n t-\cos k_{0} t\right), \forall n \in N_{\left(k_{0}\right)} .
$$

We have

$$
\begin{aligned}
\left\langle c_{n}^{\omega}, \vartheta_{k}\right\rangle & =\langle\cos n t, \cos k t\rangle-\left\langle\cos n t, \cos k_{0} t\right\rangle \\
& =\frac{\pi}{2} \delta_{n k}, \forall n, k \in N_{\left(k_{0}\right)}
\end{aligned} .
$$

On the other hand $\cos n t-\cos k_{0} t \sim t^{2}, t \rightarrow 0$, and, consequently, $\vartheta_{n}(t) \sim t^{2-\alpha_{0}}, t \rightarrow 0$. From these relations we immediately find that $\left\{\vartheta_{n}\right\}_{n \in N_{\left(k_{0}\right)}} \subset L_{q}$. Consequently, system $\left\{c_{n}^{\omega}\right\}_{n \in N_{\left(k_{0}\right)}}$ is complete and minimal in $L_{p}$. Then, it is clear that the system $\left\{c_{n}^{\omega}\right\}_{n \in Z_{+}}$has a defect equal to 1 in this case.

Consider the basicity of system $\left\{c_{n}^{\omega}\right\}_{n \in N_{\left(k_{0}\right)}}$ in $L_{p}$. By $\|\cdot\|_{p}$ we denote the ordinary norm in $L_{p}$. It is obvious that $\sup _{n}\left\|c_{n}^{\omega}\right\|_{p}<+\infty$. We have

$$
\begin{gathered}
\left\|c_{n}^{\omega}\right\|_{p}^{p}=\int_{0}^{\pi} \omega^{p}(t)|\cos n t|^{p} d t \geq \\
c_{1} \int_{0}^{\varepsilon} t^{\alpha}{ }_{0} p|\cos n t|^{p} d t=c_{2} n^{-\alpha} \int_{0}^{n \varepsilon} t^{\alpha-1}|\cos t|^{p} d t,
\end{gathered}
$$

( $c_{k}>0$ is some constant, independent of $n$ ) where the interval $[0, \varepsilon](\varepsilon>0)$ does not contain the points $\left\{t_{k}\right\}_{1}^{r}$ and $\alpha=\alpha_{0} p+1$. Assume

$$
M_{n}=\left\{k \geq 1: k \pi+\frac{\pi}{4} \leq n \varepsilon\right\} .
$$

We have

$$
\begin{gathered}
\left\|c_{n}^{\omega}\right\|_{p}^{p} \geq c_{3} n^{-\alpha} \sum_{k \in M_{n}} \int_{k \pi-\frac{\pi}{4}}^{k \pi+\frac{\pi}{4}} t^{\alpha-1}|\cos t|^{p} d t \geq \\
c_{4} n^{-\alpha} \sum_{k \in M_{n}} \int_{k \pi-\frac{\pi}{4}}^{k \pi+\frac{\pi}{4}} t^{\alpha-1} d t .
\end{gathered}
$$

It is clear that $\alpha \geq 1$, and as a result

$$
\left\|c_{n}^{\omega}\right\|_{p}^{p} \geq c_{4} n^{-\alpha} \sum_{k \in M_{n}} \int_{4 k-1}^{4 k+1} t^{\alpha-1} d t
$$

It is obvious that

$$
\int_{4 k-3}^{4 k-1} t^{\alpha-1} d t \leq \int_{4 k-1}^{4 k+1} t^{\alpha-1} d t, k \geq 1
$$

Taking the previous relations into account, from (5) we have

$$
\begin{aligned}
\left\|c_{n}^{\omega}\right\|_{p}^{p} & \geq c_{5} n^{-\alpha} \sum_{k \in M_{n}}\left[\int_{4 k-3}^{4 k-1} t^{\alpha-1} d t+\int_{4 k-1}^{4 k+1} t^{\alpha-1} d t\right], \\
& \geq c_{6} n^{-\alpha} \int_{0}^{\lambda_{n}} t^{\alpha-1} d t=c_{6}\left(\frac{\lambda_{n}}{n}\right)^{\alpha}
\end{aligned}
$$

where $\lambda_{n} \geq \frac{n \varepsilon}{\pi}-2$. Hence

$$
\left\|c_{n}^{\omega}\right\|_{p}^{p} \geq c_{6}\left(\frac{\varepsilon}{\pi}-\frac{2}{n}\right)^{\alpha} \rightarrow c_{6}\left(\frac{\varepsilon}{\pi}\right)^{\alpha}>0, n \rightarrow \infty .
$$

Consequently, it directly follows that

$$
\inf _{n}\left\|c_{n}^{\omega}\right\|_{p}>0 \text {. }
$$

Thus

$$
0<\inf _{n}\left\|c_{n}^{\omega}\right\|_{p} \leq \sup _{n}\left\|c_{n}^{\omega}\right\|_{p}<+\infty .
$$

Concerning biorthogonal system, we have

$$
\begin{gathered}
\left\|\vartheta_{n}\right\|_{q}^{q}=\int_{0}^{\pi} \omega^{-q}(t)\left|\cos n t-\cos k_{0} t\right|^{q} d t \geq \\
\geq c \int_{0}^{\varepsilon} t^{-\alpha_{0} q}\left|\cos n t-\cos k_{0} t\right|^{q} d t,
\end{gathered}
$$

where ( and in sequel also) by $c$ we'll denote positive constants, which may be different in different places. Consequently

$\left\|\vartheta_{n}\right\|_{q}^{q} \geq c\left(\int_{0}^{\varepsilon} t^{-\alpha_{0} q}|\cos n t-1|^{q} d t-\int_{0}^{\varepsilon} t^{-\alpha_{0} q}\left|\cos k_{0} t-1\right|^{q} d t\right)$.

As $\cos k_{0} t-1 \sim t^{2}, t \rightarrow 0$, then it is clear that

$$
\int_{0}^{\varepsilon} t^{-\alpha_{0} q}\left|\cos k_{0} t-1\right|^{q} d t<+\infty .
$$

Consider

$$
\begin{aligned}
I_{\mathcal{E}, n} & \equiv \int_{0}^{\varepsilon} t^{-\alpha_{0} q}|\cos n t-1|^{q} d t \\
& =n^{\alpha_{0} q-1} \int_{0}^{n \varepsilon} t^{-\alpha_{0} q}|\cos t-1|^{q} d t, \\
& =c n^{-\alpha} \int_{0}^{\frac{n \varepsilon}{2}} t^{\alpha-1} \sin ^{2 q} t d t
\end{aligned}
$$


where $\alpha=-\alpha_{0} q+1$. At first consider the case $\alpha_{0} \in\left(\frac{1}{q}, \frac{1}{q}+2\right)$, i.e. $-2 q<\alpha<0$. We have

$$
\begin{aligned}
I_{\varepsilon, n} & =c n^{-\alpha}\left(\int_{0}^{1} t^{\alpha-1} \sin ^{2 q} t d t+\int_{0}^{\frac{n \varepsilon}{2}} t^{\alpha-1} \sin ^{2 q} t d t\right) \\
& \geq c n^{-\alpha} \int_{0}^{1} t^{\alpha-1} \sin ^{2 q} t d t
\end{aligned}
$$

As, $\sin ^{2 q} t \sim t^{2 q}, t \rightarrow 0$, and $\alpha+2 q-1>-1$, then it is clear that

$$
\int_{0}^{1} t^{\alpha-1} \sin ^{2 q} t d t<+\infty
$$

and consequently, $I_{\varepsilon, n} \rightarrow+\infty, n \rightarrow \infty$. Let $\alpha_{0}=\frac{1}{q}$, i.e. $\alpha=0$. It is obvious that

$$
\int_{0}^{1} t^{-1} \sin ^{2 q} t d t<+\infty .
$$

On the other hand

$$
\begin{gathered}
\int_{1}^{n \frac{\varepsilon}{2}} t^{-1} \sin ^{2 q} d t \geq \sum_{k \in M_{1 n}} \int_{\substack{k+\frac{\pi}{4} \\
(k+1) \pi-\frac{\pi}{4}}}^{-1}|\sin t|^{2 q} d t \geq \\
c \sum_{k \in M_{n}} \int_{k \pi+\frac{\pi}{4}}^{(k+1) \pi-\frac{\pi}{4}} t^{-1} d t
\end{gathered}
$$

where $M_{n} \equiv\left\{k \geq 0:(k+1) \pi-\frac{\pi}{4} \leq n \frac{\varepsilon}{2}\right\}$. Thus

$$
I_{\varepsilon, n} \geq c \sum_{k \in M_{n}} \int_{4 k+1}^{4 k+3} t^{-1} d t .
$$

It is absolutely clear that

$$
\int_{4 k+3}^{4 k+5} t^{-1} d t \leq \int_{4 k+1}^{4 k+3} t^{-1} d t, \forall k \in M_{n} .
$$

Taking into account this relation we have

$$
\begin{aligned}
I_{\varepsilon, n} & \geq c \sum_{k \in M_{n}}\left[\int_{4 k+1}^{4 k+3} t^{-1} d t+\int_{4 k+3}^{4 k+5} t^{-1} d t\right], \\
& \geq c \int_{1}^{\lambda_{n}} t^{-1} d t=c \ln \lambda_{n}
\end{aligned}
$$

where $\lambda_{n} \geq \frac{n \varepsilon}{2 \pi}-2$. Consequently, $I_{\varepsilon, n} \rightarrow+\infty$, $n \rightarrow \infty$. As a result, we obtain $\sup _{n}\left\|\vartheta_{n}\right\|_{q}=+\infty$. Then, it is clear that $\sup _{n}\left\|c_{n}^{\omega}\right\|_{p}\left\|\vartheta_{n}\right\|_{q}=+\infty$. As a result, we get that the system $\left\{c_{n}^{\omega}\right\}_{n \in N_{\left(k_{0}\right)}}$ is not uniformly minimal in $L_{p}$ [see, for example 16] and at the same time does not form a basis for it. Let us show that in this case the system $\left\{c_{n}^{\omega}\right\}_{n \in Z_{+}}$is not a frame in $L_{p}$. Assume that it is not true. Then, it is clear that zero has a non-trivial decomposition, i.e.

$$
0=\sum_{n=0}^{\infty} a_{n} c_{n}^{\omega}
$$

It is evident that $a_{0} \neq 0$. It follows directly that the arbitrary element can be expanded with respect to the system $\left\{c_{n}^{\omega}\right\}_{n \in N_{\left(k_{0}\right)}}$. We came upon a contradiction which proves that this system does not form a basis for $L_{p}$.

Let us consider the case $\alpha_{0} \in\left[\frac{1}{q}+2, \frac{1}{q}+4\right)=M_{q}^{(2)}$. We look at the system $\left\{c_{n}^{\omega}\right\}_{n \in N_{\left\{k_{1} ; k_{2}\right\}}}$, where $N_{\left\{k_{1} ; k_{2}\right\}} \equiv Z_{+} \backslash\left\{k_{1} ; k_{2}\right\}, k_{1} ; k_{2} \in Z_{+}, k_{1} \neq k_{2} \quad$ are some numbers. Let $f \in L_{q}$ cancel this system out, that is

$$
\left\langle c_{n}^{\omega}, f\right\rangle=0, \forall n \in N_{\left\{k_{1} ; k_{2}\right\}},
$$

Using the previous reasoning, we find that for some constants $c_{1} ; c_{2}$ the following is true

$$
f(t)=\omega^{-1}(t)\left(c_{1} \cos k_{1} t+c_{2} \cos k_{2} t\right) .
$$

\section{Using representations}

$$
\cos k_{i} t=1-\frac{k_{i}^{2}}{2} t^{2}+\overline{\bar{O}}\left(t^{3}\right), t \rightarrow 0, i=1,2,
$$

we obtain

$$
f(t) \sim c_{3} t^{-\alpha_{0}} g_{1}(t)+g_{2}(t), t \rightarrow 0,
$$

( $c_{3} \neq 0$ is some constant), where

$$
g_{1}(t)=\left(c_{1}+c_{2}\right)-\frac{1}{2}\left(c_{1} k_{1}^{2}+c_{2} k_{2}^{2}\right) t^{2}
$$

and $g_{2} \in L_{q}$ is some function. Thus, $f \in L_{q}$ if and only if $t^{-\alpha_{0}} g_{1}(t) \in L_{q}$. Assume $b_{1}=\left(c_{1}+c_{2}\right), b_{2}=-\frac{1}{2}\left(c_{1} k_{1}^{2}+c_{2} k_{2}^{2}\right)$. As $-\alpha_{0} q \leq-1$ and $\left(2-\alpha_{0}\right) q \leq-1$, it is clear that $t^{-\alpha_{0}} \notin L_{q}, t^{2-\alpha_{0}} \notin L_{q}$. Suppose $b_{1} \neq 0$. We have

$$
\left|t^{-\alpha_{0}} g_{1}(t)\right|=\left|b_{1}\right| t^{-\alpha_{0}}\left|1+\frac{b_{2}}{b_{1}} t^{2}\right| \text {. }
$$

It follows directly that for sufficiently small $\delta>0$ we have 


$$
\left|t^{-\alpha_{0}} g_{1}(t)\right| \geq c_{\delta}\left|b_{1}\right| t^{-\alpha_{0}}, \forall t \in(0, \delta),
$$

where $c_{\delta} \neq 0$ is some constant. As a result $f \notin L_{q}$. Consequently, $b_{1}=0$, and it is clear that $b_{2}=0$. Thus, we obtain the following algebraic system with respect to the constants $c_{1} ; c_{2}$ :

$$
\left.\begin{array}{l}
c_{1}+c_{2}=0 \\
c_{1} k_{1}^{2}+c_{2} k_{2}^{2}=0
\end{array}\right\}
$$

It is clear that $\operatorname{det}\left|\begin{array}{cc}1 & 1 \\ k_{1}^{2} & k_{2}^{2}\end{array}\right| \neq 0$. And, consequently $c_{1}=c_{2}=0$. As a result, $f=0$, which, in turn, implies that the system $\left\{c_{n}^{\omega}\right\}_{n \in N_{\left\{k_{1} ; k_{2}\right\}}}$ is complete in $L_{p}$. Let us show that it is also minimal in $L_{p}$. Assume $\gamma_{n}^{(i)}=\frac{1}{2}\left(k_{i}^{2}-n^{2}\right)$. Consider the system

$$
\begin{aligned}
\vartheta_{n}^{(2)}(t)= & {\left[\left(\frac{1}{\gamma_{n}^{(1)}}-\frac{1}{\gamma_{n}^{(2)}}\right) \cos n t-\frac{1}{\gamma_{n}^{(1)}} \cos k_{1} t+\frac{1}{\gamma_{n}^{(2)}} \cos k_{2} t\right] } \\
& \times \omega^{-1}(t), \forall n \in N^{\left\{k_{1} ; k_{2}\right\}}
\end{aligned}
$$

We obtain directly from the following representation $\cos n t-\cos k_{i} t=\frac{1}{2}\left(k_{i}^{2}-n^{2}\right) t^{2}+\overline{\bar{O}}\left(t^{3}\right), t \rightarrow 0, i=1,2$, that $\left\{\vartheta_{n}^{(2)}\right\}_{n \in N_{\left\{k_{1} ; k_{2}\right\}}} \subset L_{q}$. On the other hand

$$
\left\langle c_{n}^{\omega}, \vartheta_{n}^{(2)}\right\rangle=\gamma_{n} \delta_{n k}, \quad \forall n, k \in N_{\left\{k_{1} ; k_{2}\right\}},
$$

where $\gamma_{n}=\frac{2}{\pi}\left(\frac{1}{\gamma_{n}^{(1)}}-\frac{1}{\gamma_{n}^{(2)}}\right) \neq 0, \forall n \in N_{\left\{k_{1} ; k_{2}\right\}}$. From these relations follows the minimality of system $\left\{c_{n}^{\omega}\right\}_{n \in N_{\left\{k_{1} ; k_{2}\right\}}}$ in $L_{p}$. Thus, if $\alpha_{0} \in M_{p}^{(2)}$, then the system $\left\{c_{n}^{\omega}\right\}_{n \in N_{\left\{k_{1} ; k_{2}\right\}}}$ is complete and minimal in $L_{p}$, and, as a result, the system $\left\{c_{n}^{\omega}\right\}_{n \in Z_{+}}$has a defect equal to 2. Continuing this way, we obtain that if $\alpha_{0} \in M_{p}^{(k)}$, where $M_{p}^{(k)} \equiv\left[\frac{1}{q}+2(k-1), \frac{1}{q}+2 k\right)$, then the system $\left\{c_{n}^{\omega}\right\}_{n \in N_{\left\{\bar{n}_{k}\right\}}}$ is complete and minimal in $L_{p}$, where $\left\{\bar{n}_{k}\right\}=\left\{n_{1} ; \ldots ; n_{k}\right\} \subset Z_{+}, n_{i} \neq n_{j}$, with $i \neq j$.

Proceeding in an absolutely similar way as we did in the previous case, we can prove that absolutely similar to the case $k=1$ we establish that for $\alpha_{0} \in M_{p}^{(k)}$ the system $\left\{c c_{n \in N_{\left\{\bar{n}_{k}\right\}}}\right.$ is not uniformly minimal in $L_{p}$, and consequently, it does not form a basis for it. Let us show that for $\alpha_{0} \in M_{p}^{(k)}, \forall k \geq 1$, the system $\left\{c_{n}^{\omega}\right\}_{n \in Z_{+}}$is not frame in $L_{p}$. Let $k=2:\left\{\bar{n}_{k}\right\} \equiv\left\{n_{1} ; n_{2}\right\}$. Assume that the system $\left\{c_{n}^{\omega}\right\}_{n \in Z_{+}}$is a frame in $L_{p}$. Then zero has a nontrivial decomposition: $0=\sum_{n=1}^{\infty} a_{n} c_{n}^{\omega}$. It is clear that $\left|a_{n_{1}}\right|+\left|a_{n_{2}}\right|>0$, and let $a_{n_{1}} \neq 0$. It follows directly that the system $\left\{c_{n}^{\omega}\right\}_{n \in N_{\left\{\bar{n}_{1}\right\}}}$ is a frame in $L_{p}$. The further reasoning is absolutely similar to the case of $k=1$. This scheme is applicable for $\forall k \in N$.

The theorem is proved.

\section{Acknowledgement}

This paper was performed under financial support of Science Foundation of the State Oil Company of Azerbaijan Republic.

The authors would like to express their profound gratitude to Prof. Bilal Bilalov, for his valuable guidance to this article.

\section{References}

[1] Babenko K.I. On conjugated functions, DAN SSSR, vol.62, No2, 1948, pp.157-160.

[2] Bari N.K. Trigonometric Series. Fizmatgiz, Moscow, 1961, 672 p.

[3] Bilalov B.T., Veliyev S.G. On completeness of exponent system with complex coefficients in weight spaces. Trans. of NAS of Azerb., XXV, No 7, 2005, p. 9 - 14.

[4] Bilalov B.T., Veliyev S.G. Bases of eigen functions of two discontinuous differential operators. Diff. Uravn. 2006, vol. 42, No 9, p. 190-192.

[5] Christensen O. An Introduction to Frames and Riesz bases. Springer, 2003, $440 \mathrm{p}$

[6] Edwards R. Fourier Series in a Modern Exposition. Moscow, "Mir", vol.1, 1985, 264 p.

[7] Edwards R. Fourier Series in a Modern Exposition. Moscow, "Mir", vol.2, 1985, $400 \mathrm{p}$.

[8] Gaposhkin V.F. One generalization of M.Riesz theorem on conjugated functions. Mat. Sbornik, 46 (3) (88), 1958, 111-115.

[9] Gar J. Garnett. Bounded Analytic Functions. Moscow, "Mir", $1984,469 \mathrm{p}$.

[10] Gohberg I.C., Krein M.G. Introduction to the theory of linear nonselfadjoint operators. Nauka", Moscow 1965448 p.

[11] Golubeva E.S. The System of Weighted Exponentials with Power Weights. Herald of Samara State University. Natural Sciences, 2011, No2 (83), pp.15-25.

[12] Bilalov B.T., Guliyeva F.A. On The Frame Properties of Degenerate System of Sines, Journal of Function Spaces and Applications, Volume 2012 (2012), Article ID 184186, 12 pages.

[13] Heil Ch. A Basis Theory Primer, Springer, 2011, 534 p.

[14] Kazaryan K.S., Lizorkin P.I. Multipliers, bases and unconditional bases in the weighted spaces B and SB, Proc. of the Steklov Ins. of Math., 1989, 187, pp. 111-130.

[15] Moiseev E.I. On basicity of systems of cosines and sines in weight space. Diff. Uravn., vol. 34, No.1,1998,pp. 40-44.

[16] Moiseev E.I. The basicity in the weight space of a system of eigen functions of a differential operator. Diff. Uravn., 1999, v.35, No2, pp.200-205.

[17] Pukhov S.S., Sedletskii A.M. Bases of exponents, sines and cosines in weight spaces on finite interval. Dokl. RAN vol. 425,4 (2009), pp.452-455.

[18] Zygmund A. Trigonometric series. Moscow, "Mir", vol.1, 1965, 616p.

[19] Zygmund A. Trigonometric series. Moscow, "Mir", vol.2, 1965, 538 p. 